\title{
The Oxidative Pentose Phosphate Pathway is the Primary Source of NADPH for Lipid Overproduction from Glucose in Yarrowia lipolytica
}

\author{
Thomas M. Wasylenko, Woo Suk Ahn, and Gregory Stephanopoulos \\ Department of Chemical Engineering, Massachusetts Institute of Technology, \\ Cambridge, MA 02139, USA
}

Email addresses: Thomas M. Wasylenko: twasylen@mit.edu

Woo Suk Ahn:wsahn@mit.edu

Gregory Stephanopoulos: gregstep@ mit.edu

Correspondence: Prof. Gregory Stephanopoulos

Dept. of Chemical Engineering

Massachusetts Institute of Technology

Room 56-469C

77 Massachusetts Ave

Cambridge, MA 02139

Phone: (617) 253-4583

Fax: (617) 253-3122

Email: gregstep@mit.edu 


\begin{abstract}
Oleaginous microbes represent an attractive means of converting a diverse range of feedstocks into oils that can be transesterified to biodiesel. However, the mechanism of lipid overproduction in these organisms is incompletely understood, hindering the development of strategies for engineering superior biocatalysts for "single-cell oil" production. In particular, it is unclear which pathways are used to generate the large quantities of NADPH required for overproduction of the highly reduced fatty acid species. While early studies implicated malic enzyme as having a key role in production of lipogenic NADPH in oleaginous fungi, several recent reports have cast doubts as to whether malic enzyme may contribute to production of lipogenic NADPH in the model oleaginous yeast Yarrowia lipolytica. To address this problem we have used ${ }^{13} \mathrm{C}$-Metabolic Flux Analysis to estimate the metabolic flux distributions during lipid accumulation in two $Y$. lipolytica strains; a control strain and a previously published engineered strain capable of producing lipids at roughly twice the yield. We observe a dramatic rearrangement of the metabolic flux distribution in the engineered strain which supports lipid overproduction. The NADPH-producing flux through the oxidative Pentose Phosphate Pathway is approximately doubled in the engineered strain in response to the roughly two-fold increase in fatty acid biosynthesis, while the flux through malic enzyme does not differ significantly between the two strains. Moreover, the estimated rate of NADPH production in the oxidative Pentose Phosphate Pathway is in good agreement with the estimated rate of NADPH consumption in fatty acid biosynthesis in both strains. These results suggest the oxidative Pentose Phosphate Pathway is the primary source of lipogenic NADPH in Y. lipolytica.
\end{abstract}




\section{Keywords}

${ }^{13}$ C-Metabolic Flux Analysis; Biodiesel; NADPH; Oleaginous Yeast; Pentose Phosphate Pathway; Yarrowia lipolytica 


\section{Introduction}

In recent years, concerns about the sustainability and environmental impact of fossil fuels have given impetus to research into alternative sources of liquid fuels for the transportation industry. Biodiesel has emerged as a promising biofuel and is currently produced predominantly by transesterification of vegetable oils derived from rapeseed, palm, and soybean, with a small fraction being produced from waste oils (Gui et al., 2008). However, given the finite supply of arable land and increasing demand for food, it is unlikely that food-based biofuels will contribute significantly to meeting future transportation energy needs (Hill et al., 2006). It has been proposed that biodiesel could be produced utilizing oleaginous microbes capable of accumulating lipids produced from a wide range of feedstocks (Li et al., 2008; Meng et al., 2009; Ageitos et al., 2011). Such a process for production of "single-cell oil" would have several advantages over current biodiesel production technologies. The process would be independent of climate and variations in weather (e.g. drought), and production facilities could be located on non-arable land so as to not compete directly with the food supply (Beopoulos et al., 2011). Most importantly, land productivity in the case of carbohydrate production and conversion to oil is much higher than can be achieved by direct production of oil crops. Economical production of biodiesel using oleaginous microbes will require optimization of the biocatalysts, which can be achieved through metabolic engineering (Bailey, 1991). The oleaginous yeast Yarrowia lipolytica has emerged as both a model oleaginous organism and a promising host for single-cell oil production because of the availability of genome sequence data (Dujon et al., 2004) and welldeveloped tools for genetic engineering (Beopoulos et al., 2009). Although much work has been done to elucidate the mechanism of lipid accumulation in oleaginous microbes (Ratledge, 2002), 
to guide the engineering of $Y$. lipolytica strains which can accumulate lipids at high yield, titer, and productivity a more comprehensive understanding will be required.

High lipid accumulation in oleaginous microbes is typically achieved with nitrogen-limiting culture conditions. After nitrogen is depleted from the growth medium, cells can no longer synthesize nucleotides and proteins. Consequently, oleaginous microbes begin to convert the excess carbon source remaining in the medium to lipids, which are generally stored as triacylglycerols (TAGs). It is believed that in many oleaginous organisms lipid accumulation is initiated by a decrease in the intracellular adenosine monophosphate (AMP) pool (Boulton and Ratledge, 1983), which may be mediated by AMP deaminase (which converts AMP to inosine monophosphate and ammonium in order to scavenge for nitrogen) (Evans and Ratledge, 1985; Wynn et al., 2001) or may simply result from a decreased demand for ATP upon cessation of nucleotide and protein synthesis (Marchal et al., 1977). AMP is an allosteric regulator of mitochondrial isocitrate dehydrogenase (IDH) (Mitsushima et al., 1978; Botham and Ratledge, 1979), and the resultant decrease in IDH activity causes an accumulation of isocitrate and citrate (which may be rapidly interconverted by aconitase) in the mitochondria. The accumulated citrate is transported out to the cytosol (usually the mechanism is citrate-malate antiport) (Evans et al., 1983a, 1983b), where it is acted upon by ATP citrate lyase, generating a molecule of oxaloacetate and a molecule of acetyl-CoA (AcCoA) (Boulton and Ratledge, 1981). The AcCoA is the substrate for fatty acid biosynthesis. The oxaloacetate can be converted to malate by cytosolic malate dehydrogenase (MDH). As one molecule of malate is transported into the mitochondria in exchange for citrate, the malate can be converted back to oxaloacetate by mitochondrial MDH and condense with a mitochondrial AcCoA two-carbon unit (catalyzed by 
citrate synthase) to regenerate mitochondrial citrate. Thus, the net effect of this process is the shuttling of one molecule of AcCoA from the mitochondria to the cytosol (and one NADH reducing equivalent from the cytosol into the mitochondria), fueling lipid overproduction (Evans et al., 1983a). The cycle for shuttling AcCoA from the mitochondria to the cytosol for fatty acid synthesis is depicted in Fig. 1.

Because lipids are highly reduced species, TAG overproduction also requires large quantities of NADPH, with two NADPH being oxidized to $\mathrm{NADP}^{+}$for each fatty acid elongation step and one NADPH being consumed for each fatty acid desaturation reaction. It is unclear how oleaginous microbes meet this high NADPH demand during lipid accumulation. There is evidence that malic enzyme plays a major role in providing lipogenic NADPH in the oleaginous fungi Mucor circinelloides and Mortierella alpina (Wynn et al., 1997, 1999), and overexpression of malic enzyme in $M$. circinelloides resulted in a 2.5 -fold increase in lipid content and an increase in the degree of unsaturation of accumulated fatty acids (Zhang et al., 2007). Overexpression of the $M$. circinelloides malic enzyme in Rhodotorula glutinis also increased lipid content more than twofold (Z. Li et al., 2013). A recent study found that malic enzyme activity increased significantly in Y. lipolytica after the onset of lipid accumulation (Ochoa-Estopier and Guillouet, 2014). However, the single malic enzyme detected in the $Y$. lipolytica genome is predicted to localize to the mitochondria, while lipid accumulation requires generation of NADPH in the cytosol, where lipid biosynthesis occurs (Beopoulos et al., 2011). Moreover, an in vitro study has shown that the Y. lipolytica malic enzyme prefers $\mathrm{NAD}^{+}$to $\mathrm{NADP}^{+}$(Zhang et al., 2013). Taken together, these results suggest malic enzyme does not play a meaningful role in providing reducing power for lipid accumulation in $Y$. lipolytica. Indeed, a transcriptomic study showed that malic enzyme expression did not change significantly upon depletion of nitrogen from the growth medium 
(Morin et al., 2011), and overexpression of neither the endogenous malic enzyme (Beopoulos et al., 2011) nor the NADP ${ }^{+}$-dependent malic enzyme from M. alpina (Zhang et al., 2013) had a significant effect on lipid accumulation in this oleaginous yeast. These results have led to speculation that the oxidative Pentose Phosphate Pathway (PPP) is the primary source of lipogenic NADPH in Y. lipolytica (Zhang et al., 2013; Tai and Stephanopoulos, 2013).

In order to investigate the impact of lipid overproduction on central carbon metabolism, we have conducted ${ }^{13}$ C-Metabolic Flux Analysis (MFA) (Wiechert, 2001) on a previously engineered $Y$. lipolytica strain overexpressing acetyl-CoA carboxylase and diacylglycerol acyltransferase, which is capable of producing lipids from glucose at high yield and to high final lipid content (Tai and Stephanopoulos, 2013). Precise flux estimates were obtained by fitting data from

parallel labeling experiments with two complementary ${ }^{13} \mathrm{C}$-glucose tracers (Crown and Antoniewicz, 2013). The results indicate the oxidative PPP is the primary source of lipogenic NADPH in $Y$. lipolytica overproducing TAGs from glucose.

\section{Materials and Methods}

\subsection{Strains and Culture Conditions}

Experiments were conducted with two previously described $Y$. lipolytica strains: a control strain MTYL037 and a lipid-overproducing strain MTYL065 which overexpresses ACC1 (encoding acetyl-CoA carboxylase) and DGAl (encoding diacylglycerol acyltransferase) (Tai and Stephanopoulos, 2013). Strains were maintained at $4{ }^{\circ} \mathrm{C}$ on Yeast Extract-Peptone-Dextrose (YPD) plates and cultured at $30{ }^{\circ} \mathrm{C}$ in minimal media containing $20 \mathrm{~g} / 1$ glucose as the sole carbon source, ammonium sulfate as the sole nitrogen source, and $1.7 \mathrm{~g} / \mathrm{l}$ Yeast Nitrogen Base 
without amino acids and ammonium sulfate (Amresco, Solon, $\mathrm{OH}$ ) as a source of salts, vitamins, and trace elements. All cultures were shaken at $250 \mathrm{rpm}$.

Initially a single $50 \mathrm{ml}$ shake flask starter culture of each strain was inoculated from the corresponding YPD plate. The starter culture medium contained $5 \mathrm{~g} / \mathrm{l}$ ammonium sulfate, resulting in an initial carbon/nitrogen $(\mathrm{C} / \mathrm{N})$ ratio of 8.8 . After approximately $24 \mathrm{~h}$, each starter culture was used to inoculate three shake flask cultures. The media for these cultures contained $0.44 \mathrm{~g} / \mathrm{l}$ ammonium sulfate, resulting in an initial $\mathrm{C} / \mathrm{N}$ ratio of 100 . For each strain, each of the three shake flask cultures contained a different glucose substrate: (1) glucose labeled to natural abundance; (2) $1,2-{ }^{13} \mathrm{C}_{2}$-glucose (Omicron Biochemicals, South Bend, IN); and (3) $20 \% \mathrm{U}_{-}{ }^{13} \mathrm{C}_{6^{-}}$ glucose (Cambridge Isotope Laboratories, Tewksbury, MA). The three cultures were otherwise identical.

Cells were washed prior to inoculation in order to remove residual starter culture medium and prevent carryover of unlabeled glucose from the starter cultures to the cultures containing ${ }^{13} \mathrm{C}$ labeled glucose substrates. $1 \mathrm{ml}$ starter culture was centrifuged $5 \mathrm{~min}$ at maximum speed $(18,000 \mathrm{~g})$ and the supernatant discarded. The cell pellet was resuspended in $1 \mathrm{ml}$ of the medium to be used in the labeling experiment (e.g. the cell pellet to be used for inoculation of a $1,2{ }^{13} \mathrm{C}_{2^{-}}$ glucose culture would have been resuspended in $1,2-{ }^{13} \mathrm{C}_{2}$-glucose medium), centrifuged a second time, and the supernatant discarded. Finally, cells were resuspended in $1 \mathrm{ml}$ of the medium to be used in the labeling experiment and transferred to the $50 \mathrm{ml}$ shake flask culture. 


\subsection{Extracellular Metabolite Quantification}

Extracellular glucose, citrate, mannitol, and erythritol concentrations were measured by HighPerformance Liquid Chromatography (HPLC). Cells were removed from culture samples by filtration through $0.20 \mu \mathrm{m}$ Nylon syringe filters (Microliter Analytical \#F13-2020-1GF). HPLC was performed with a Waters 2695 Separation Module coupled to a Waters 410 Differential Refractometer. Metabolite separation was achieved on an Aminex HPX-87H column (Bio-Rad, Hercules, CA) with an isocratic flow of $14 \mathrm{mM}$ sulfuric acid mobile phase at $0.7 \mathrm{ml} / \mathrm{min}$ and a temperature of $50{ }^{\circ} \mathrm{C}$. The injection volume was $10 \mu 1$.

Extracellular ammonium was quantified using a commercially available Ammonia Assay Kit (Sigma-Aldrich, \#AA0100).

\subsection{Dry Cell Weight Measurements}

Cell densities were monitored by measuring dry cell weight (DCW). $1 \mathrm{ml}$ culture was vacuumfiltered on a pre-weighed cellulose nitrate filter $(0.2 \mu \mathrm{m}$, Whatman \#10401312). Filters were washed with two volumes Millipore water, dried at $60{ }^{\circ} \mathrm{C}$, and weighed intermittently until the mass no longer changed over time. At each time point two control filters were prepared by filtering $1 \mathrm{ml}$ cell-free natural abundance glucose medium. The control filters were used to correct for the losses in filter mass that occur during sample preparation.

\subsection{Lipid Analysis}

The five principal fatty acid species synthesized by $Y$. lipolytica (palmitate, C16:0; palmitoleate, C16:1; stearate, C18:0; oleate, C18:1; linoleate, C18:2) were quantified using a Gas 
Chromatography-Flame Ionization Detector (GC-FID) setup. No other fatty acid species were detected in significant quantities. Depending on cell density, 0.7-1 $\mathrm{ml}$ cell culture was centrifuged $10 \mathrm{~min}$ at $18,000 \mathrm{~g}$ and the supernatant removed. The volume of cell culture was chosen so that each sample would contain roughly $2-3 \mathrm{mg}$ biomass (as measured by DCW). Cell pellets were stored at $-20{ }^{\circ} \mathrm{C}$ until derivatization. For lipid analysis, $100 \mu 1$ internal standard solution (2 $\mathrm{mg} / \mathrm{ml}$ methyl tridecanoate (Sigma-Aldrich \#T0627) $+2 \mathrm{mg} / \mathrm{ml}$ glyceryl triheptadecanoate (Sigma-Aldrich \#T2151) in hexane) was added to each cell pellet. Methyl tridecanoate was used to correct for volume losses during sample preparation and glyceryl triheptadecanoate was used to correct for transesterification efficiency. Lipids were transesterified to fatty acid methyl esters (FAMEs) by addition of $500 \mu \mathrm{l} 0.5 \mathrm{~N}$ sodium methoxide (20 g/l sodium hydroxide in methanol) followed by 60 min vortexing at $1200 \mathrm{rpm}$. Samples were then neutralized by addition of $40 \mu 1$ sulfuric acid. FAMEs were extracted by addition of $500 \mu \mathrm{l}$ hexane followed by another 30 min vortexing at $1200 \mathrm{rpm}$. Samples were centrifuged $1 \mathrm{~min}$ at $8000 \mathrm{rpm}$ (roughly 6000g) and the hexane layer was subsequently analyzed on a Bruker 450-GC Gas Chromatograph equipped with a FID. Samples were injected with an injection volume of $1 \mu \mathrm{l}$, split ratio of 10 , and injector temperature of $260{ }^{\circ} \mathrm{C}$. FAME species were separated on an Agilent J\&W HP-INNOWax capillary column ( $30 \mathrm{~m}$ x $0.25 \mathrm{~mm} \times 0.15 \mu \mathrm{m}$, \#19091N-033) with helium carrier gas at a flow rate of $1.5 \mathrm{ml} / \mathrm{min}$. The column oven was held at a constant temperature of $200{ }^{\circ} \mathrm{C}$ for the duration of the method. The FID was operated at a temperature of $260{ }^{\circ} \mathrm{C}$ with a helium make up gas flow of $25 \mathrm{ml} / \mathrm{min}$, hydrogen flow of 30 $\mathrm{ml} / \mathrm{min}$, and air flow of $300 \mathrm{ml} / \mathrm{min}$. 


\subsection{Estimation of Extracellular Fluxes}

The GC-FID lipid data were used to calculate the total moles of AcCoA and glycerol-3phosphate (Glyc3P) consumed for lipid biosynthesis by assuming that one mole AcCoA was consumed for every two moles of carbon incorporated into fatty acids and that all lipids were in the form of TAGs and therefore one mole Glyc3P was required for every three moles of fatty acids produced. The total NADPH consumption for lipid biosynthesis was also calculated by assuming that two NADPH were required for each fatty acid elongation step and one NADPH was required for each fatty acid desaturation step. However, no mass balance constraint on NADPH was included in the metabolic model, as inclusion of such redox cofactor balances may introduce significant errors due to assumptions about enzyme cofactor preferences and possible omission of important sources or sinks from the metabolic model (Marx et al., 1996; Schmidt et al., 1998). Consequently, the NADPH consumption calculations were not used in the metabolic flux estimation. The AcCoA, Glyc3P, and NADPH requirements for synthesis of each of the five principal fatty acids produced by $Y$. lipolytica are summarized in Table 1 .

\begin{tabular}{lccc} 
Fatty Acid & AcCoA & Glyc3P & NADPH \\
\hline Palmitate (C16:0) & 8 & 0.333 & 14 \\
Palmitoleate (C16:1) & 8 & 0.333 & 15 \\
Stearate (C18:0) & 9 & 0.333 & 16 \\
Oleate (C18:1) & 9 & 0.333 & 17 \\
Linoleate (C18:2) & 9 & 0.333 & 18
\end{tabular}

Table 1 Acetyl-CoA, Glycerol-3-Phosphate, and NADPH requirements for biosynthesis of each of the five principal fatty acids in $Y$. lipolytica 
All metabolic fluxes were normalized to a glucose uptake rate of 100 . For each culture, the molar concentrations of citrate, mannitol, and erythritol and the molar consumption of lipogenic AcCoA, Glyc3P, and NADPH were plotted against the molar glucose concentration and the slope of each best-fit line was computed using the Microsoft Excel function LINEST. These slopes were then multiplied by 100 to determine the moles of citrate, mannitol, and erythritol produced and the moles of lipogenic AcCoA, Glyc3P, and NADPH consumed per 100 moles of glucose taken up. For each strain, the results from each of the three shake flask cultures were averaged and the standard deviations were computed as estimates of the uncertainties in the computed extracellular flux values.

\subsection{Metabolite Extractions}

$7.5 \mathrm{ml}$ culture was added to $37.5 \mathrm{ml}$ pure methanol quenching solution (Canelas et al., 2008) held at low temperature $\left(<-70{ }^{\circ} \mathrm{C}\right)$ in a cold ethanol bath. Cells were pelleted by centrifugation for 5 min at $3270 \mathrm{~g}$ and $-10{ }^{\circ} \mathrm{C}$ and the supernatant removed by aspiration. Cells were washed by resuspension in $40 \mathrm{ml}$ cold $\left(<-70^{\circ} \mathrm{C}\right)$ methanol followed by centrifugation and aspiration of the supernatant (as before). Metabolites were extracted from quenched cell pellets in $5 \mathrm{ml}$ hot (80 $\left.{ }^{\circ} \mathrm{C}\right) 75 \%$ (v/v) ethanol extraction solvent (Gonzalez et al., 1997; Canelas et al., 2009). Samples were vortexed for $30 \mathrm{~s}$, held at $80{ }^{\circ} \mathrm{C}$ in a water bath for $3 \mathrm{~min}$, vortexed again for $30 \mathrm{~s}$, cooled briefly in the cold ethanol bath, and centrifuged to clear cell debris. Each metabolite extract was split into two equal fractions (each roughly $2.5 \mathrm{ml}$ ) for GC-MS and LC-MS/MS analysis, respectively, and stored at $-80^{\circ} \mathrm{C}$. 


\subsection{Gas Chromatography-Mass Spectrometry (GC-MS) Analysis of Metabolite Extracts}

Metabolite extracts were dried under airflow using a Pierce Reacti-Therm III Heating/Stirring Module and resuspended in $20 \mu \mathrm{l} 2 \%$ methoxyamine-hydrogen chloride in pyridine (Methoxamine (MOX) Reagent, Thermo Scientific \#TS-45950). The methoximation reaction was allowed to proceed for $90 \mathrm{~min}$ at $37{ }^{\circ} \mathrm{C}$. Subsequently a silylation reaction was performed by addition of $25 \mu \mathrm{l}$ N-tert-Butyldimethylsilyl-N-methyltrifluoroacetamide with $1 \%$ tertButyldimethylchlorosilane (Sigma-Aldrich, \#375934) to each sample and incubation at $60{ }^{\circ} \mathrm{C}$ for 30 min. Samples were centrifuged 2 min to remove cell debris and the supernatants analyzed on an Agilent 6890N Network GC System coupled to an Agilent 5975B Inert XL MSD. The injection volume was $3 \mu 1$. The GC-MS conditions have been described elsewhere (Wasylenko and Stephanopoulos, 2014). Mass spectra were acquired in Selective Ion Monitoring (SIM) mode (Ahn and Antoniewicz, 2013).

\subsection{Liquid Chromatography-Tandem Mass Spectrometry (LC-MS/MS)}

Metabolite extracts were dried under airflow using a Pierce Reacti-Therm III Heating/Stirring Module, resuspended in $80 \mu \mathrm{l}$ Millipore water, and analyzed on an Agilent 1100 Series HPLC system coupled to an API 2000 MS/MS (AB Sciex, Framingham, MA). The injection volume was $20 \mu \mathrm{l}$. Metabolites were separated on a Waters XBridge C18 Column (2.1 mm x $150 \mathrm{~mm}$, $130 \AA ̊ ., 3.5 \mu \mathrm{m}$; \#186003023) using an ion pair chromatography method adapted from (Luo et al., 2007; Young et al., 2011). The HPLC flow rate was $300 \mu 1 / \mathrm{min}$ and the solvent gradient was:

0\% B (0 min), 0\% B (8 min), 22.5\% B (18 min), 40\% B (28 min), 60\% B (32 min), 90\% B (34 $\min ), 90 \% \mathrm{~B}(36 \mathrm{~min}), 100 \% \mathrm{~B}(37 \mathrm{~min}), 100 \% \mathrm{~B}(42 \mathrm{~min})$, where A is $10 \mathrm{mM}$ tributylamine + $15 \mathrm{mM}$ acetic acid in water and B is methanol. Mass spectra were acquired in multiple reaction 
monitoring (MRM) mode.

\subsection{Gas Chromatography-Mass Spectrometry (GC-MS) Analysis of Glucose}

The isotopic purities of $1,2-{ }^{13} \mathrm{C}_{2}$ - and $\mathrm{U}_{-}{ }^{13} \mathrm{C}_{6}$-glucose tracers were determined by GC-MS analysis of aldonitrile pentapropionate glucose derivatives (Antoniewicz et al., 2011). $5 \mu 1$ fresh glucose medium was dried using a Pierce Reacti-Therm III Heating/Stirring Module. Dry residues were dissolved in $50 \mu \mathrm{l}$ pyridine solution containing $20 \mathrm{mg} / \mathrm{ml}$ hydroxylamine hydrochloride and incubated at $90{ }^{\circ} \mathrm{C}$ for $60 \mathrm{~min} .100 \mu \mathrm{l}$ propionic anhydride was added to each sample, followed by incubation at $60{ }^{\circ} \mathrm{C}$ for an additional $30 \mathrm{~min}$. Samples were dried, resuspended in $100 \mu \mathrm{l}$ ethyl acetate, and centrifuged to remove insolubles. The supernatants were then analyzed by GC-MS. The injection volume was $1 \mu 1$ with split ratio 9:1, and the inlet temperature was $250{ }^{\circ} \mathrm{C}$. The helium carrier gas flow rate was $0.9 \mathrm{ml} / \mathrm{min}$. The $\mathrm{GC}$ column oven temperature gradient was: $80{ }^{\circ} \mathrm{C}(0 \mathrm{~min}), 80^{\circ} \mathrm{C}(1 \mathrm{~min}), 280{ }^{\circ} \mathrm{C}(11 \mathrm{~min}), 280{ }^{\circ} \mathrm{C}(15 \mathrm{~min})$. Mass spectra were acquired by scanning the range $150-450 \mathrm{~m} / \mathrm{z}$. All other details of the GC-MS method were identical to those for analysis of metabolite extracts. Glucose tracer isotopic purities were estimated from ${ }^{13} \mathrm{C}$-labeling of fragments at $259 \mathrm{~m} / \mathrm{z}\left(\mathrm{U}_{-}{ }^{13} \mathrm{C}_{6}\right.$-glucose only) and 284 and $370 \mathrm{~m} / \mathrm{z}$ (both tracers) (Antoniewicz et al., 2011).

\subsection{Metabolic Flux Estimation}

The metabolic fluxes through a compartmentalized model reaction network were computed by nonlinear parameter estimation. The model network comprised the reactions of the Glycolysis, PPP, and Tricarboxylic Acid (TCA) Cycle pathways as well as pathways for production of extracellular citrate, mannitol, and erythritol and incorporation of AcCoA and Glyc3P into 
TAGs. Cytosolic pyruvate carboxylase (Otto et al., 2012) and mitochondrial malic enzyme (Beopoulos et al., 2011) reactions were also included. The non-oxidative PPP was modeled using the half-reaction method of (Kleijn et al., 2005). The model contained separate cytosolic and mitochondrial pools of AcCoA, citrate, malate, oxaloacetate, and pyruvate. For malate, oxaloacetate, and pyruvate, the ${ }^{13} \mathrm{C}$-labeling patterns of the cytosolic and mitochondrial pools are not necessarily the same. For each of these "compartmentalized" metabolites, both the cytosolic and mitochondrial pools were allowed to contribute to the ${ }^{13} \mathrm{C}$-labeling data, with the contribution of each pool left as a free parameter in the model. Pyruvate was assumed to be transported unidirectionally from the cytosol to the mitochondria, as has been assumed for Saccharomyces cerevisiae (Maaheimo et al., 2001). Citrate was assumed to be transported across the mitochondrial membrane in exchange with malate (Evans et al., 1983a, 1983b). Anaplerosis was assumed to be mediated by irreversible transport of malate from the cytosol to the mitochondria via the dicarboxylate carrier (Palmieri et al., 1996; Luévano-Martínez et al., 2010), likely in exchange for phosphate and indirectly driven by the proton gradient across the mitochondrial membrane (Palmieri et al., 2000). Inclusion of either an oxaloacetate carrier reaction (Palmieri et al., 1999; Luévano-Martínez et al., 2010) or a reversible malate transport reaction did not have any significant effect on the flux estimation, and these reactions were therefore excluded. Of the gluconeogenic enzymes, PEP carboxykinase was omitted from the model while fructose-1,6-bisphosphatase was included, as the former is glucose-repressed (albeit incompletely) in Y. lipolytica while the latter is not (Jardón et al., 2008). The glyoxylate shunt was also omitted from the reaction network. Although this pathway is only incompletely repressed on glucose in Y. lipolytica (Flores and Gancedo, 2005; Jardón et al., 2008), Blank, et al. found no evidence of flux through the pathway in $Y$. lipolytica during growth on glucose 
(Blank et al., 2005). Consistent with these results, we found that the estimated flux through the glyoxylate shunt was negligible when the pathway was included in the model (data not shown). The complete model reaction network with atom transitions can be found in Supplementary Table S1.

Metabolic flux estimation for each strain was achieved by generating a random initial flux distribution, using the model network to simulate the expected extracellular flux and metabolite labeling data corresponding to this flux distribution, and then iteratively refining the flux distribution to minimize the lack-of-fit (as measured by the weighted sum of squared residuals) between the simulated data and the data obtained in the carbon labeling experiments. The flux estimation for each strain was repeated 500 times with different initial guesses and the smallest weighted sum of squared residuals obtained taken to be the global optimum. The best-fit flux distributions were subjected to a chi-square test for goodness-of-fit, and $68 \%$ and $95 \%$ confidence intervals were calculated for each flux using a parameter continuation technique (Antoniewicz et al., 2006). The uncertainties in the ${ }^{13} \mathrm{C}$-labeling data mass isotopomer mole fractions were assumed to be $0.4 \mathrm{~mol} \%$ for the goodness-of-fit test and confidence interval determination (Antoniewicz et al., 2007a; Wasylenko and Stephanopoulos, 2013). All computations were performed with an in-house software that formulates the simulation problem in terms of elementary metabolite units (EMUs) (Antoniewicz et al., 2007b). The expected effects of naturally occurring heavy isotopes were added to simulated data, and the corrected simulated data were compared directly to experiment data (Wittmann and Heinzle, 1999; van Winden et al., 2002). Reversible reactions were modeled as two fluxes, a net flux and an exchange flux (Wiechert and de Graaf, 1997). 


\section{Results}

\subsection{Fermentation Profiles and Establishment of Metabolic Steady State}

We compared the performances of the engineered lipid-overproducing strain MTYL065 and the control strain MTYL037 in a low-nitrogen medium that promotes lipid accumulation (initial C/N ratio $=100$ ). Each strain was cultured in triplicate, and for each strain one replicate was cultured in $1,2-{ }^{13} \mathrm{C}_{2}$-glucose medium and another in $20 \% \mathrm{U}_{-}{ }^{13} \mathrm{C}_{6}$-glucose medium to facilitate ${ }^{13} \mathrm{C}-\mathrm{MFA}$. The third replicate culture for each strain contained glucose labeled to natural abundance. The fermentation profiles for the six shake flask cultures are shown in Figs. 2 and 3. The engineered strain MTYL065 consumed glucose and produced lipids more rapidly than the control strain MTYL037. The three MTYL065 cultures achieved final lipid contents of 57-59\% compared to only $22-26 \%$ for MTYL037. The final lipid profiles of the two strains were similar (Fig. 4), with oleate and palmitate being the most abundant fatty acid species and accounting for roughly $50 \%$ and $25 \%$ of the total lipids, respectively. The only by-products detected by HPLC were citrate, mannitol, and erythritol. The two strains produced mannitol at similar rates and titers, although, since MTYL065 consumed glucose more rapidly, its mannitol yield was significantly lower than that of MTYL037. The engineered strain produced small amounts of citrate during the initial phase of the fermentation, but in the latter stages (after the ammonium supply was exhausted) citrate production was negligible. The control strain continued to produce citrate even after the ammonium in the medium was depleted. Both strains produced only small amounts of erythritol.

In conventional ${ }^{13} \mathrm{C}-\mathrm{MFA}$, it is assumed that intracellular metabolite labeling patterns are at steady state. To satisfy this assumption, cultures must be maintained in a "metabolic steady state" where the metabolic fluxes are invariant in time until the intracellular metabolite ${ }^{13} \mathrm{C}$ labeling patterns reach "isotopic steady state," at which point metabolites are harvested 
(Wiechert and de Graaf, 1997; Wiechert, 2001). Labeling data for this study were obtained from primary central carbon metabolites directly rather than from biomass macromolecule hydrolysates. Due to the fast turnover times of these central carbon metabolites, if a culture can be maintained in metabolic steady state the metabolite ${ }^{13} \mathrm{C}$-labeling patterns are expected to reach isotopic steady state relatively quickly (van Winden et al., 2005; Canelas et al., 2008).

Metabolic steady state conditions can be achieved in chemostat cultures, and in batch cultures it is generally assumed that exponential growth phase approximates a metabolic steady state. In a $Y$. lipolytica culture with a high initial $\mathrm{C} / \mathrm{N}$ ratio, the cells are expected to grow roughly exponentially until the ammonium nitrogen source is depleted from the medium. At this point, the cells can no longer produce nucleotide and protein biomass and lipid accumulation commences. This lipid accumulation phase is more relevant than exponential growth phase for lipid overproduction; however it was not obvious that the cultures would be in a metabolic steady state during this phase.

If the cultures were to reach a steady state during the lipid accumulation phase, the number of cells would be expected to be roughly constant due to the absence of nitrogen in the growth medium. A constant number of cells with constant metabolic fluxes would be expected to generate metabolite profiles which vary linearly in time. We observed that by $40 \mathrm{~h}$ the ammonium supply had been exhausted in all cultures (Fig. 2) and between 40 and $64 \mathrm{~h}$ all cultures exhibited roughly linear glucose consumption and lipid, citrate, mannitol, and erythritol production profiles (Figs. 2 and 3). Thus we concluded that between 40 and $64 \mathrm{~h}$ the cultures were in lipid accumulation phase and could be assumed to approximate a metabolic steady state. 
The metabolic flux estimation was performed using data acquired during this $24 \mathrm{~h}$ timeframe, which is sufficiently long that the central carbon metabolites could be expected to be at isotopic steady state at the end of the experiment.

\subsection{Estimation of Extracellular Fluxes}

The glucose uptake rate was fixed to an arbitrary value of 100 for all cultures to facilitate comparison of the intracellular metabolic flux distributions in the two strains. The other extracellular fluxes were then determined from the yields of citrate, mannitol, erythritol, and lipogenic AcCoA, Glyc3P, and NADPH per 100 mol glucose, which were calculated by modeling the HPLC and GC-FID data acquired between 40 and $64 \mathrm{~h}$ through linear regression (see Materials and Methods). The data used for the linear regressions and the resulting best-fit lines are shown in Supplementary Figs. S1-S6. The extracellular flux values and their uncertainties used in metabolic flux estimation are listed in Table 2. It can be seen that the lipid yield achieved by the engineered strain was more than two-fold higher than that of the control. The control strain produced all three by-products citrate, mannitol, and erythritol at higher yield than the engineered strain.

\begin{tabular}{lcc} 
& MTYL037 & MTYL065 \\
\cline { 2 - 3 } Glucose & 100 & 100 \\
Citrate & $2.1 \pm 0.7$ & 0 \\
Mannitol & $6.9 \pm 0.9$ & $3.6 \pm 0.5$ \\
Erythritol & $0.47 \pm 0.03$ & $0.24 \pm 0.03$ \\
AcCoA & $56 \pm 4$ & $124 \pm 3$ \\
Glyc3P & $2.2 \pm 0.2$ & $4.75 \pm 0.09$ \\
NADPH & $104 \pm 8$ & $228 \pm 5$
\end{tabular}

Table 2 Extracellular flux values used for ${ }^{13} \mathrm{C}-\mathrm{MFA}$ 


\subsection{Metabolic Flux Estimation}

${ }^{13}$ C-MFA was performed on strains MTYL037 and MTYL065 using the estimated extracellular flux values and GC-MS and LC-MS/MS metabolite labeling data as inputs. GC-MS analysis of the glucose tracers confirmed that each tracer had an isotopic purity of approximately $99 \%$, consistent with manufacturer specifications. The best-fit metabolic flux distributions are shown in Fig. 5, and the confidence intervals for key fluxes are shown in Fig. 6. The weighted sums of squared residuals for the MTYL037 and MTYL065 flux estimation models were 198.9 and 165.9 , respectively. These values fell within the range that would be expected given that models adequately describe the data, which was 145.6 to 220.2 . The measured and simulated ${ }^{13} \mathrm{C}$ labeling data from the flux estimation algorithm are presented in Supplementary Table S2, and the full set of best-fit flux values and flux confidence intervals are tabulated in Supplementary Table S3.

The computed metabolic flux distributions reveal that the flux of AcCoA to lipids in the engineered strain MTYL065 is increased more than twofold over the control strain, and there is a dramatic rearrangement of the metabolic flux distribution to support lipid-overproduction. The majority of mitochondrial citrate produced by citrate synthase is exported to the cytosol as a shuttle for lipogenic AcCoA. Consequently, the flux through isocitrate dehydrogenase is decreased by roughly $70 \%$, although there is still significant flux through the TCA Cycle. The fluxes through ATP citrate lyase and cytosolic malate dehydrogenase are also increased to support the shuttling of AcCoA across the mitochondrial membrane. Malate is transported from the cytosol to the mitochondria in exchange for citrate (and also by the dicarboxylate carrier.) 
However, the most striking feature of the metabolic flux distributions is the large increase in flux through the oxidative PPP in the engineered strain.

In the control strain MTYL037 the flux through the oxidative PPP is estimated to be 52.0 (Fig. 5). As 2 moles of NADPH are produced for each mole of glucose-6-phosphate (G6P) metabolized through this pathway, the total NADPH production by the oxidative PPP is estimated to be 104 moles per 100 moles glucose consumed. This number is in perfect agreement with the estimate for NADPH consumed in fatty acid biosynthesis (Table 2). (Note that an NADPH mass balance was not included in the metabolic network, so the matching of the rates of NADPH consumption and production is non-trivial.) Thus the flux through the oxidative PPP appears to be sufficient to supply all of the NADPH required for lipid production in MTYL037.

In the engineered strain MTYL065, the flux of AcCoA to TAG biosynthesis is increased roughly two-fold (from 59.8 to 123.3). The flux through the oxidative PPP is also increased by roughly a factor of two, from 52.0 to 102.2. In fact, the flux through the oxidative PPP exceeds the glucose uptake flux; the flux through phosphoglucose isomerase is reversed with a small net flux from fructose-6-phosphate supplying the G6P substrate for the oxidative PPP. In this strain, the predicted NADPH production rate of 204.4 is in good agreement with the estimated NADPH requirement for fatty acid biosynthesis, which is 228 (Table 2), although there is a roughly $10 \%$ gap between the rates of NADPH production and consumption. This gap could result from experimental error or could indicate that an alternative source of NADPH not included in the 
model contributes to NADPH production in MTYL065. Regardless, the oxidative PPP appears to supply the majority of the reducing power required for fatty acid biosynthesis in this strain.

The flux confidence intervals in Fig. 6 show that the flux through the oxidative PPP was estimated with good resolution in both strains. The correlation between the flux of AcCoA to lipids and the oxidative PPP flux is clear. However, the flux through malic enzyme was not significantly different in the two strains, suggesting that malic enzyme does not play a significant role in lipid production in $Y$. lipolytica.

The increase in flux through the oxidative PPP in MTYL065 is evident from the ${ }^{13} \mathrm{C}$-labeling data. The mass isotopomer distributions (MIDs) of several key metabolites from the $1,2-{ }^{13} \mathrm{C}_{2^{-}}$ glucose labeling experiment are shown in Fig. 7. With this tracer, the majority of the carbon atoms lost as $\mathrm{CO}_{2}$ in the 6-phosphogluconate dehydrogenase reaction will be ${ }^{13} \mathrm{C}$. Consequently, increased flux through the oxidative PPP will result in increased abundance of light mass isotopomers and decreased abundance of heavy mass isotopomers in the MIDs. G6P and 6phosphogluconate (6PG) must in theory have identical ${ }^{13} \mathrm{C}$-labeling patterns, since $6 \mathrm{PG}$ is derived from G6P via a linear pathway. The MIDs of G6P and 6PG are in good agreement for both strains. It can be seen that the relative abundances of the lighter $M+1$ and $M+2$ mass isotopomers are higher in MTYL065 than in MTYL037 while the relative abundances of the heavier mass isotopomers $(\mathrm{M}+3$ and higher) are lower, reflecting the increased oxidative PPP flux in the engineered strain. Dihydroxyacetone phosphate (DHAP) and 3-phosphoglycerate (3PG) are also expected to have similar ${ }^{13} \mathrm{C}$-labeling patterns due to the high exchange flux of the triosephosphate isomerase reaction (Fig. 5), although the DHAP MID is skewed by the presence 
of derivatization groups added to facilitate GC-MS analysis. Nonetheless, it can be seen that in both the DHAP and 3PG MIDs the relative abundances of the M+0 and M+1 mass isopotomers are higher in MTYL065 than in MTYL037 while the relative abundances of the heavier mass isotopmers (M+2 and higher) are lower, providing further confirmation that the oxidative PPP flux is elevated in the engineered strain.

\section{Discussion}

We have conducted ${ }^{13} \mathrm{C}$-MFA of two $Y$. lipolytica strains during lipid accumulation on glucose. High flux resolution was achieved by combining ${ }^{13} \mathrm{C}$-labeling data from parallel labeling experiments utilizing two different ${ }^{13} \mathrm{C}$-glucose tracers, $1,2-{ }^{13} \mathrm{C}_{2}$-glucose and $20 \% \mathrm{U}_{-}{ }^{13} \mathrm{C}_{6}$ glucose (Crown and Antoniewicz, 2013). The ${ }^{13} \mathrm{C}$-labeling data imply that there is a dramatic increase in flux through the oxidative PPP in the lipid-overproducing strain MTYL065 relative to the control strain MTYL037. Indeed, quantitative metabolic flux estimation revealed that the NADPHgenerating flux through the oxidative PPP is comparable to the NADPH-consuming flux of AcCoA to fatty acid biosynthesis in both strains. The flux through malic enzyme was similar in both strains and did not correlate with lipid production. Taken together, these results indicate that the oxidative PPP is likely the primary source of lipogenic NADPH during lipid overproduction from glucose in $Y$. lipolytica.

As noted above, an NADPH mass balance constraint was not included in the metabolic model; the model contained no information about production or consumption of NADPH equivalents. Therefore the result that the estimated flux through the NADPH-producing reactions of the oxidative PPP increases roughly proportionally to the NADPH-consuming flux of AcCoA to lipids in MTYL065 is non-trivial. With the modeling approach used, it would have been 
mathematically possible for the flux of AcCoA to lipids to increase without any increase in flux through NADPH-producing reactions. The flux of AcCoA to lipids in each strain is determined largely by the HPLC and GC-FID data (see Table 2). Conversely, the increase in flux through the oxidative PPP in MTYL065 reflects differences in the metabolite ${ }^{13} \mathrm{C}$-labeling patterns of the two strains (see Fig. 7). Thus the conclusion that the oxidative PPP and fatty acid biosynthesis fluxes are correlated in the two $Y$. lipolytica strains investigated here comes directly from the HPLC, GC-FID, and ${ }^{13} \mathrm{C}$-labeling data and is not dependent on any modeling assumptions about NADPH production and consumption.

The utilization of the oxidative PPP to supply NADPH for fatty acid biosynthesis may not be unique to $Y$. lipolytica among oleaginous yeasts; upregulation of 6-phosphogluconate dehydrogenase during lipid accumulation has previously been observed in proteomic studies of Rhodosporidium toruloides and Lipomyces starkeyi (Liu et al., 2009, 2011). The heavy usage of the oxidative PPP for NADPH generation is suboptimal for production of lipids from glucose at high yields, as one mole of carbon is lost as carbon dioxide for every two moles of NADPH produced. A recent analysis showed that higher lipid yields could be achieved if NADPH were produced from cytosolic NADH generated in glycolysis (e.g. by a transhydrogenase cycle mediated by pyruvate carboxylase, malate dehydrogenase, and cytosolic NADP-dependent malic enzyme) or from mitochondrial NADH (e.g. by a transhydrogenase cycle consisting of mitochondrial $\mathrm{NAD}^{+}$-dependent IDH and cytosolic $\mathrm{NADP}^{+}$-dependent IDH operating in opposite directions) without net generation of carbon dioxide (Ratledge, 2014). In S. cerevisiae, cytosolic $\mathrm{NADP}^{+}$-dependent IDH and a citrate/ $\alpha-$ ketoglutarate antiporter apparently play an important role in supplying cytosolic NADPH (Castegna et al., 2010). However, Y. lipolytica possesses only a 
single NADP ${ }^{+}$-dependent IDH, which likely localizes to the mitochondria (X. Li et al., 2013). The malic enzyme transhydrogenase cycle is apparently intimately related to lipid overproduction in other oleaginous organisms (Wynn et al., 1997, 1999; Zhang et al., 2007), but not $Y$. lipolytica (Beopoulos et al., 2011; Zhang et al., 2013). The observed production of the sugar alcohols mannitol and erthyritol, which are formed by reduction of mannose and erythrose respectively, suggests that under the conditions investigated here the cell does indeed have an excess supply of cytosolic NADH which must be re-oxidized to $\mathrm{NAD}^{+}$and could therefore be used to generate NADPH equivalents. Engineering of pathways for production of NADPH without loss of carbon represents a promising avenue for future research.

In sum, the overexpression of $A C C 1$ and DGA1 in MTYL065 drastically increases the rate of lipid synthesis in this strain, roughly doubling the demand for NADPH. To satisfy this increased demand, fluxes are redistributed at the G6P node such as to increase flux through the oxidative PPP. There does not appear to be any regulation preventing this redistribution; thus in $Y$. lipolytica G6P is likely a flexible branch point (Stephanopoulos and Vallino, 1991) at which the partitioning of flux readily changes to meet requirements for NADPH production. An increased rate of lipid production may feedback to the G6P node by increasing the rate of NADPH oxidation, resulting in a larger fraction of G6P being metabolized through the oxidative PPP to meet the increased demand for lipogenic NADPH. However, this mechanism is suboptimal from a yield perspective, and lipid yields could potentially benefit from increasing the supply of NADPH through mechanisms which do not involve net loss of carbon through decarboxylation reactions. 


\section{Acknowledgements}

The authors thank Kangjian Qiao and Andrew Silverman for many helpful discussions. This work was supported by the United States Department of Energy's Genomic Science research program grant number DE-SC0008744.

\section{References}

Ageitos, J.M., Vallejo, J.A., Veiga-Crespo, P., Villa, T.G., 2011. Oily yeasts as oleaginous cell factories. Appl. Microbiol. Biotechnol. 90, 1219-1227. doi:10.1007/s00253-011-3200-z

Ahn, W.S., Antoniewicz, M.R., 2013. Parallel labeling experiments with [1,2-(13)C]glucose and [U-(13)C]glutamine provide new insights into CHO cell metabolism. Metab. Eng. 15, 34-47. doi:10.1016/j.ymben.2012.10.001

Antoniewicz, M.R., Kelleher, J.K., Stephanopoulos, G., 2006. Determination of confidence intervals of metabolic fluxes estimated from stable isotope measurements. Metab. Eng. 8, 324-337. doi:10.1016/j.ymben.2006.01.004

Antoniewicz, M.R., Kelleher, J.K., Stephanopoulos, G., 2007a. Accurate assessment of amino acid mass isotopomer distributions for metabolic flux analysis. Anal. Chem. 79, 75547559. doi:10.1021/ac0708893

Antoniewicz, M.R., Kelleher, J.K., Stephanopoulos, G., 2007b. Elementary metabolite units (EMU): a novel framework for modeling isotopic distributions. Metab. Eng. 9, 68-86. doi:10.1016/j.ymben.2006.09.001 
Antoniewicz, M.R., Kelleher, J.K., Stephanopoulos, G., 2011. Measuring deuterium enrichment of glucose hydrogen atoms by gas chromatography/mass spectrometry. Anal. Chem. 83, 3211-3216. doi:10.1021/ac200012p

Bailey, J.E., 1991. Toward a science of metabolic engineering. Science 252, 1668-1675.

Beopoulos, A., Cescut, J., Haddouche, R., Uribelarrea, J.-L., Molina-Jouve, C., Nicaud, J.-M., 2009. Yarrowia lipolytica as a model for bio-oil production. Prog. Lipid Res. 48, 375387. doi:10.1016/j.plipres.2009.08.005

Beopoulos, A., Nicaud, J.-M., Gaillardin, C., 2011. An overview of lipid metabolism in yeasts and its impact on biotechnological processes. Appl. Microbiol. Biotechnol. 90, 11931206. doi:10.1007/s00253-011-3212-8

Blank, L.M., Lehmbeck, F., Sauer, U., 2005. Metabolic-flux and network analysis in fourteen hemiascomycetous yeasts. FEMS Yeast Res. 5, 545-558.

doi:10.1016/j.femsyr.2004.09.008

Botham, P.A., Ratledge, C., 1979. A biochemical explanation for lipid accumulation in Candida 107 and other oleaginous micro-organisms. J. Gen. Microbiol. 114, 361-375.

Boulton, C.A., Ratledge, C., 1981. Correlation of Lipid Accumulation in Yeasts with Possession of ATP: Citrate Lyase. Microbiology 127, 169-176. doi:10.1099/00221287-127-1-169

Boulton, C.A., Ratledge, C., 1983. Use of Transition Studies in Continuous Cultures of Lipomyces starkeyi, an oleaginous yeast, to Investigate the Physiology of Lipid Accumulation. Microbiology 129, 2871-2876. doi:10.1099/00221287-129-9-2871

Canelas, A.B., Ras, C., Pierick, A., Dam, J.C., Heijnen, J.J., Gulik, W.M., 2008. Leakage-free rapid quenching technique for yeast metabolomics. Metabolomics 4, 226-239. doi:10.1007/s11306-008-0116-4 
Canelas, A.B., ten Pierick, A., Ras, C., Seifar, R.M., van Dam, J.C., van Gulik, W.M., Heijnen, J.J., 2009. Quantitative evaluation of intracellular metabolite extraction techniques for yeast metabolomics. Anal. Chem. 81, 7379-7389. doi:10.1021/ac900999t

Castegna, A., Scarcia, P., Agrimi, G., Palmieri, L., Rottensteiner, H., Spera, I., Germinario, L., Palmieri, F., 2010. Identification and functional characterization of a novel mitochondrial carrier for citrate and oxoglutarate in Saccharomyces cerevisiae. J. Biol. Chem. 285, 17359-17370. doi:10.1074/jbc.M109.097188

Crown, S.B., Antoniewicz, M.R., 2013. Parallel labeling experiments and metabolic flux analysis: Past, present and future methodologies. Metab. Eng. 16, 21-32. doi:10.1016/j.ymben.2012.11.010

Dujon, B., Sherman, D., Fischer, G., Durrens, P., Casaregola, S., Lafontaine, I., De Montigny, J., Marck, C., Neuvéglise, C., Talla, E., Goffard, N., Frangeul, L., Aigle, M., Anthouard, V., Babour, A., Barbe, V., Barnay, S., Blanchin, S., Beckerich, J.-M., Beyne, E., Bleykasten, C., Boisramé, A., Boyer, J., Cattolico, L., Confanioleri, F., De Daruvar, A., Despons, L., Fabre, E., Fairhead, C., Ferry-Dumazet, H., Groppi, A., Hantraye, F., Hennequin, C., Jauniaux, N., Joyet, P., Kachouri, R., Kerrest, A., Koszul, R., Lemaire, M., Lesur, I., Ma, L., Muller, H., Nicaud, J.-M., Nikolski, M., Oztas, S., Ozier-Kalogeropoulos, O., Pellenz, S., Potier, S., Richard, G.-F., Straub, M.-L., Suleau, A., Swennen, D., Tekaia, F., Wésolowski-Louvel, M., Westhof, E., Wirth, B., Zeniou-Meyer, M., Zivanovic, I., Bolotin-Fukuhara, M., Thierry, A., Bouchier, C., Caudron, B., Scarpelli, C., Gaillardin, C., Weissenbach, J., Wincker, P., Souciet, J.-L., 2004. Genome evolution in yeasts. Nature 430, 35-44. doi:10.1038/nature02579 
Evans, C.T., Ratledge, C., 1985. Possible regulatory roles of ATP:citrate lyase, malic enzyme, and AMP deaminase in lipid accumulation by Rhodosporidium toruloides CBS 14. Can. J. Microbiol. 31, 1000-1005. doi:10.1139/m85-189

Evans, C.T., Scragg, A.H., Ratledge, C., 1983a. A comparative study of citrate efflux from mitochondria of oleaginous and non-oleaginous yeasts. Eur. J. Biochem. FEBS 130, 195204.

Evans, C.T., Scragg, A.H., Ratledge, C., 1983b. Regulation of citrate efflux from mitochondria of oleaginous and non-oleaginous yeasts by adenine nucleotides. Eur. J. Biochem. FEBS $132,609-615$.

Flores, C.-L., Gancedo, C., 2005. Yarrowia lipolytica mutants devoid of pyruvate carboxylase activity show an unusual growth phenotype. Eukaryot. Cell 4, 356-364. doi:10.1128/EC.4.2.356-364.2005

Gonzalez, B., François, J., Renaud, M., 1997. A rapid and reliable method for metabolite extraction in yeast using boiling buffered ethanol. Yeast Chichester Engl. 13, 1347-1355. doi:10.1002/(SICI)1097-0061(199711)13:14<1347::AID-YEA176>3.0.CO;2-O

Gui, M.M., Lee, K.T., Bhatia, S., 2008. Feasibility of edible oil vs. non-edible oil vs. waste edible oil as biodiesel feedstock. Energy 33, 1646-1653. doi:10.1016/j.energy.2008.06.002

Hill, J., Nelson, E., Tilman, D., Polasky, S., Tiffany, D., 2006. Environmental, economic, and energetic costs and benefits of biodiesel and ethanol biofuels. Proc. Natl. Acad. Sci. U. S. A. 103, 11206-11210. doi:10.1073/pnas.0604600103 
Jardón, R., Gancedo, C., Flores, C.-L., 2008. The gluconeogenic enzyme fructose-1,6bisphosphatase is dispensable for growth of the yeast Yarrowia lipolytica in gluconeogenic substrates. Eukaryot. Cell 7, 1742-1749. doi:10.1128/EC.00169-08

Kleijn, R.J., van Winden, W.A., van Gulik, W.M., Heijnen, J.J., 2005. Revisiting the 13C-label distribution of the non-oxidative branch of the pentose phosphate pathway based upon kinetic and genetic evidence. FEBS J. 272, 4970-4982. doi:10.1111/j.17424658.2005.04907.x

Li, Q., Du, W., Liu, D., 2008. Perspectives of microbial oils for biodiesel production. Appl. Microbiol. Biotechnol. 80, 749-756. doi:10.1007/s00253-008-1625-9

Li, X., Wang, P., Ge, Y., Wang, W., Abbas, A., Zhu, G., 2013. NADP(+)-specific isocitrate dehydrogenase from oleaginous yeast Yarrowia lipolytica CLIB122: biochemical characterization and coenzyme sites evaluation. Appl. Biochem. Biotechnol. 171, 403416. doi:10.1007/s12010-013-0373-1

Li, Z., Sun, H., Mo, X., Li, X., Xu, B., Tian, P., 2013. Overexpression of malic enzyme (ME) of Mucor circinelloides improved lipid accumulation in engineered Rhodotorula glutinis. Appl. Microbiol. Biotechnol. 97, 4927-4936. doi:10.1007/s00253-012-4571-5

Liu, H., Zhao, X., Wang, F., Jiang, X., Zhang, S., Ye, M., Zhao, Z.K., Zou, H., 2011. The proteome analysis of oleaginous yeast Lipomyces starkeyi. FEMS Yeast Res. 11, 42-51. doi:10.1111/j.1567-1364.2010.00687.x

Liu, H., Zhao, X., Wang, F., Li, Y., Jiang, X., Ye, M., Zhao, Z.K., Zou, H., 2009. Comparative proteomic analysis of Rhodosporidium toruloides during lipid accumulation. Yeast Chichester Engl. 26, 553-566. doi:10.1002/yea.1706 
Luévano-Martínez, L.A., Moyano, E., de Lacoba, M.G., Rial, E., Uribe-Carvajal, S., 2010. Identification of the mitochondrial carrier that provides Yarrowia lipolytica with a fatty acid-induced and nucleotide-sensitive uncoupling protein-like activity. Biochim. Biophys. Acta 1797, 81-88. doi:10.1016/j.bbabio.2009.09.003

Luo, B., Groenke, K., Takors, R., Wandrey, C., Oldiges, M., 2007. Simultaneous determination of multiple intracellular metabolites in glycolysis, pentose phosphate pathway and tricarboxylic acid cycle by liquid chromatography-mass spectrometry. J. Chromatogr. A 1147, 153-164. doi:10.1016/j.chroma.2007.02.034

Maaheimo, H., Fiaux, J., Cakar, Z.P., Bailey, J.E., Sauer, U., Szyperski, T., 2001. Central carbon metabolism of Saccharomyces cerevisiae explored by biosynthetic fractional (13)C labeling of common amino acids. Eur. J. Biochem. FEBS 268, 2464-2479.

Marchal, R., Vandecasteele, J.P., Metche, M., 1977. Regulation of the central metabolism in relation to citric acid production in Saccharomycopsis lipolytica. Arch. Microbiol. 113, 99-104.

Marx, A., de Graaf, A.A., Wiechert, W., Eggeling, L., Sahm, H., 1996. Determination of the fluxes in the central metabolism of Corynebacterium glutamicum by nuclear magnetic resonance spectroscopy combined with metabolite balancing. Biotechnol. Bioeng. 49, 111-129. doi:10.1002/(SICI)1097-0290(19960120)49:2<111::AID-BIT1>3.0.CO;2-T

Meng, X., Yang, J., Xu, X., Zhang, L., Nie, Q., Xian, M., 2009. Biodiesel production from oleaginous microorganisms. Renew. Energy 34, 1-5. doi:10.1016/j.renene.2008.04.014

Mitsushima, K., Shinmyo, A., Enatsu, T., 1978. Control of citrate and 2-oxoglutarate formation in Candida lipolytica mitochondria by adenine nucleotides. Biochim. Biophys. Acta 538, $481-492$. 
Morin, N., Cescut, J., Beopoulos, A., Lelandais, G., Le Berre, V., Uribelarrea, J.-L., MolinaJouve, C., Nicaud, J.-M., 2011. Transcriptomic analyses during the transition from biomass production to lipid accumulation in the oleaginous yeast Yarrowia lipolytica. PloS One 6, e27966. doi:10.1371/journal.pone.0027966

Ochoa-Estopier, A., Guillouet, S.E., 2014. D-stat culture for studying the metabolic shifts from oxidative metabolism to lipid accumulation and citric acid production in Yarrowia lipolytica. J. Biotechnol. 170, 35-41. doi:10.1016/j.jbiotec.2013.11.008

Otto, C., Yovkova, V., Aurich, A., Mauersberger, S., Barth, G., 2012. Variation of the byproduct spectrum during $\alpha$-ketoglutaric acid production from raw glycerol by overexpression of fumarase and pyruvate carboxylase genes in Yarrowia lipolytica. Appl. Microbiol. Biotechnol. 95, 905-917. doi:10.1007/s00253-012-4085-1

Palmieri, L., Palmieri, F., Runswick, M.J., Walker, J.E., 1996. Identification by bacterial expression and functional reconstitution of the yeast genomic sequence encoding the mitochondrial dicarboxylate carrier protein. FEBS Lett. 399, 299-302.

Palmieri, L., Runswick, M.J., Fiermonte, G., Walker, J.E., Palmieri, F., 2000. Yeast mitochondrial carriers: bacterial expression, biochemical identification and metabolic significance. J. Bioenerg. Biomembr. 32, 67-77.

Palmieri, L., Vozza, A., Agrimi, G., De Marco, V., Runswick, M.J., Palmieri, F., Walker, J.E., 1999. Identification of the yeast mitochondrial transporter for oxaloacetate and sulfate. J. Biol. Chem. 274, 22184-22190.

Ratledge, C., 2002. Regulation of lipid accumulation in oleaginous micro-organisms. Biochem. Soc. Trans. 30, 1047-1050. doi:10.1042/ 
Ratledge, C., 2014. The role of malic enzyme as the provider of NADPH in oleaginous microorganisms: a reappraisal and unsolved problems. Biotechnol. Lett. doi:10.1007/s10529-014-1532-3

Schmidt, Marx, de Graaf AA, Wiechert, Sahm, Nielsen, Villadsen, 1998. 13C tracer experiments and metabolite balancing for metabolic flux analysis: comparing two approaches. Biotechnol. Bioeng. 58, 254-257.

Stephanopoulos, G., Vallino, J.J., 1991. Network rigidity and metabolic engineering in metabolite overproduction. Science 252, 1675-1681.

Tai, M., Stephanopoulos, G., 2013. Engineering the push and pull of lipid biosynthesis in oleaginous yeast Yarrowia lipolytica for biofuel production. Metab. Eng. 15, 1-9. doi:10.1016/j.ymben.2012.08.007

Van Winden, W.A., van Dam, J.C., Ras, C., Kleijn, R.J., Vinke, J.L., van Gulik, W.M., Heijnen, J.J., 2005. Metabolic-flux analysis of Saccharomyces cerevisiae CEN.PK113-7D based on mass isotopomer measurements of (13)C-labeled primary metabolites. FEMS Yeast Res. 5, 559-568. doi:10.1016/j.femsyr.2004.10.007

Van Winden, W.A., Wittmann, C., Heinzle, E., Heijnen, J.J., 2002. Correcting mass isotopomer distributions for naturally occurring isotopes. Biotechnol. Bioeng. 80, 477-479. doi:10.1002/bit.10393

Wasylenko, T.M., Stephanopoulos, G., 2013. Kinetic isotope effects significantly influence intracellular metabolite (13) C labeling patterns and flux determination. Biotechnol. J. 8, 1080-1089. doi:10.1002/biot.201200276 
Wasylenko, T.M., Stephanopoulos, G., 2014. Metabolomic and (13) C-metabolic flux analysis of a xylose-consuming Saccharomyces cerevisiae strain expressing xylose isomerase. Biotechnol. Bioeng. doi:10.1002/bit.25447

Wiechert, W., 2001. 13C Metabolic Flux Analysis. Metab. Eng. 3, 195-206. doi:10.1006/mben.2001.0187

Wiechert, W., de Graaf, A.A., 1997. Bidirectional reaction steps in metabolic networks: I. Modeling and simulation of carbon isotope labeling experiments. Biotechnol. Bioeng. 55, 101-117. doi:10.1002/(SICI)1097-0290(19970705)55:1<101::AID-BIT12>3.0.CO;2-P

Wittmann, Heinzle, 1999. Mass spectrometry for metabolic flux analysis. Biotechnol. Bioeng. $62,739-750$.

Wynn, J.P., bin Abdul Hamid, A., Ratledge, C., 1999. The role of malic enzyme in the regulation of lipid accumulation in filamentous fungi. Microbiol. Read. Engl. 145 ( Pt 8), 19111917.

Wynn, J.P., Hamid, A.A., Li, Y., Ratledge, C., 2001. Biochemical events leading to the diversion of carbon into storage lipids in the oleaginous fungi Mucor circinelloides and Mortierella alpina. Microbiol. Read. Engl. 147, 2857-2864.

Wynn, J.P., Kendrick, A., Ratledge, C., 1997. Sesamol as an inhibitor of growth and lipid metabolism in Mucor circinelloides via its action on malic enzyme. Lipids 32, 605-610.

Young, J.D., Shastri, A.A., Stephanopoulos, G., Morgan, J.A., 2011. Mapping photoautotrophic metabolism with isotopically nonstationary (13)C flux analysis. Metab. Eng. 13, 656665. doi:10.1016/j.ymben.2011.08.002

Zhang, H., Zhang, L., Chen, H., Chen, Y.Q., Ratledge, C., Song, Y., Chen, W., 2013. Regulatory properties of malic enzyme in the oleaginous yeast, Yarrowia lipolytica, and its non- 
involvement in lipid accumulation. Biotechnol. Lett. 35, 2091-2098.

doi:10.1007/s10529-013-1302-7

Zhang, Y., Adams, I.P., Ratledge, C., 2007. Malic enzyme: the controlling activity for lipid production? Overexpression of malic enzyme in Mucor circinelloides leads to a 2.5 -fold increase in lipid accumulation. Microbiol. Read. Engl. 153, 2013-2025.

doi:10.1099/mic.0.2006/002683-0 


\section{Figure Legends}

Fig. 1. Cycle for shuttling acetyl-CoA from the mitochondria to the cytosol for fatty acid synthesis. Citrate acts as the carrier for acetyl-CoA two-carbon units. The cycle also results in transport of one NADH equivalent from the cytosol to the mitochondria and consumes one ATP in the ATP citrate lyase reaction.

Fig. 2. Extracellular metabolite fermentation profiles. Time courses for glucose and ammonium consumption and dry cell weight, citrate, mannitol, and erythritol accumulation in shake flask cultures of strains MTYL037 and MTYL065. Abbreviations denoting glucose substrate for each culture: NA, natural abundance glucose; U, 20\% U- ${ }^{13} \mathrm{C}_{6}$-glucose; $12,1,2-{ }^{13} \mathrm{C}_{2}$-glucose.

Fig. 3. Fatty acid fermentation profiles. Time courses for total lipid titer and total lipogenic AcCoA, Glyc3P, and NADPH consumed for TAG biosynthesis in shake flask cultures of strains MTYL037 and MTYL065. Abbreviations denoting glucose substrate for each culture: NA, natural abundance glucose; $\mathrm{U}, 20 \% \mathrm{U}_{-}{ }^{13} \mathrm{C}_{6}$-glucose; $12,1,2-{ }^{13} \mathrm{C}_{2}$-glucose.

Fig. 4. Final fatty acid distribution profiles. The relative abundances of the five principal fatty acid species in strains MTYL037 and MTYL065 at the time cells were harvested for ${ }^{13}$ C-MFA. 
Fig. 5. Estimated metabolic flux distributions. Two flux values are listed for each reaction; these are the fluxes in the control strain MTYL037 (top) and the engineered strain MTYL065 (bottom). Each reversible reaction is described by the net flux with the exchange flux listed inside parentheses. Exchange fluxes denoted "nr" could not be resolved within one order of magnitude.

Fig. 6. Select flux confidence intervals. Confidence intervals are shown for fluxes which illustrate the key differences between the flux distributions in strains MTYL037 and MTYL065. $68 \%$ confidence intervals are indicated by boxes and $95 \%$ confidence intervals by error bars ("whiskers"). The line inside each box indicates the best-fit flux value. All fluxes are normalized to a glucose uptake rate of 100 .

Fig. 7. Mass isotopomer distributions (MIDs) of illustrative metabolites from the $1,2{ }^{13} \mathrm{C}_{2^{-}}$ glucose labeling experiment. The relative abundances (i.e. mole fractions) of the various mass isotopomers of 6-phosphogluconate (6PG), glucose-6-phosphate (G6P), 3-phosphoglycerate (3PG), and dihydroxyacetone phosphate (DHAP) in strains MTYL037 and MTYL065 are shown. 6PG, G6P, and 3PG were measured by LC-MS/MS, DHAP by GC-MS. 


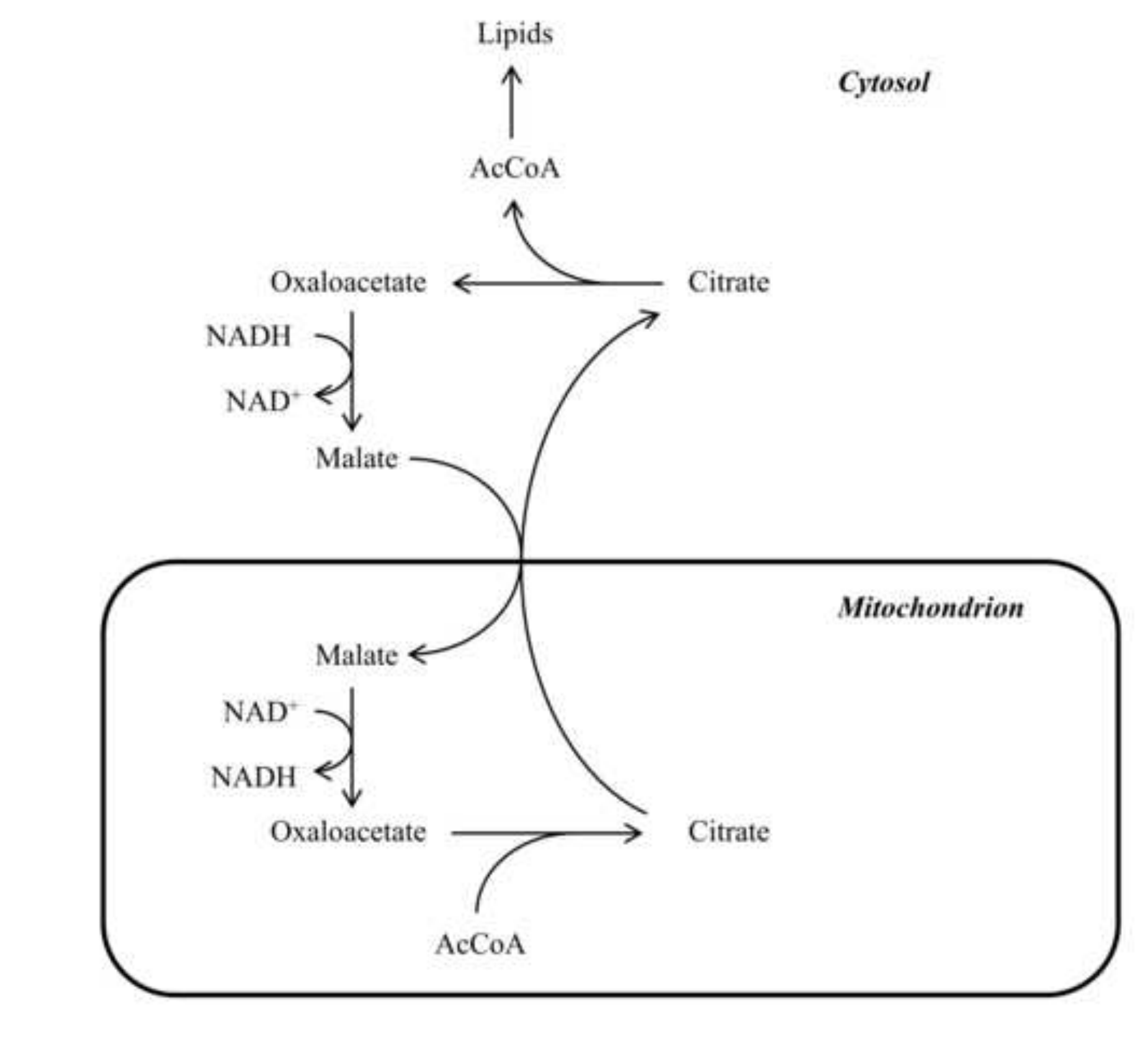

Figure 1

Lipids

.

\section{Figure}
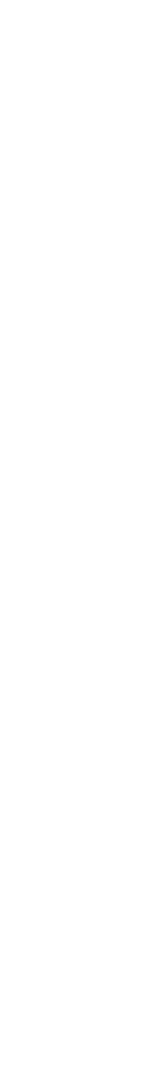

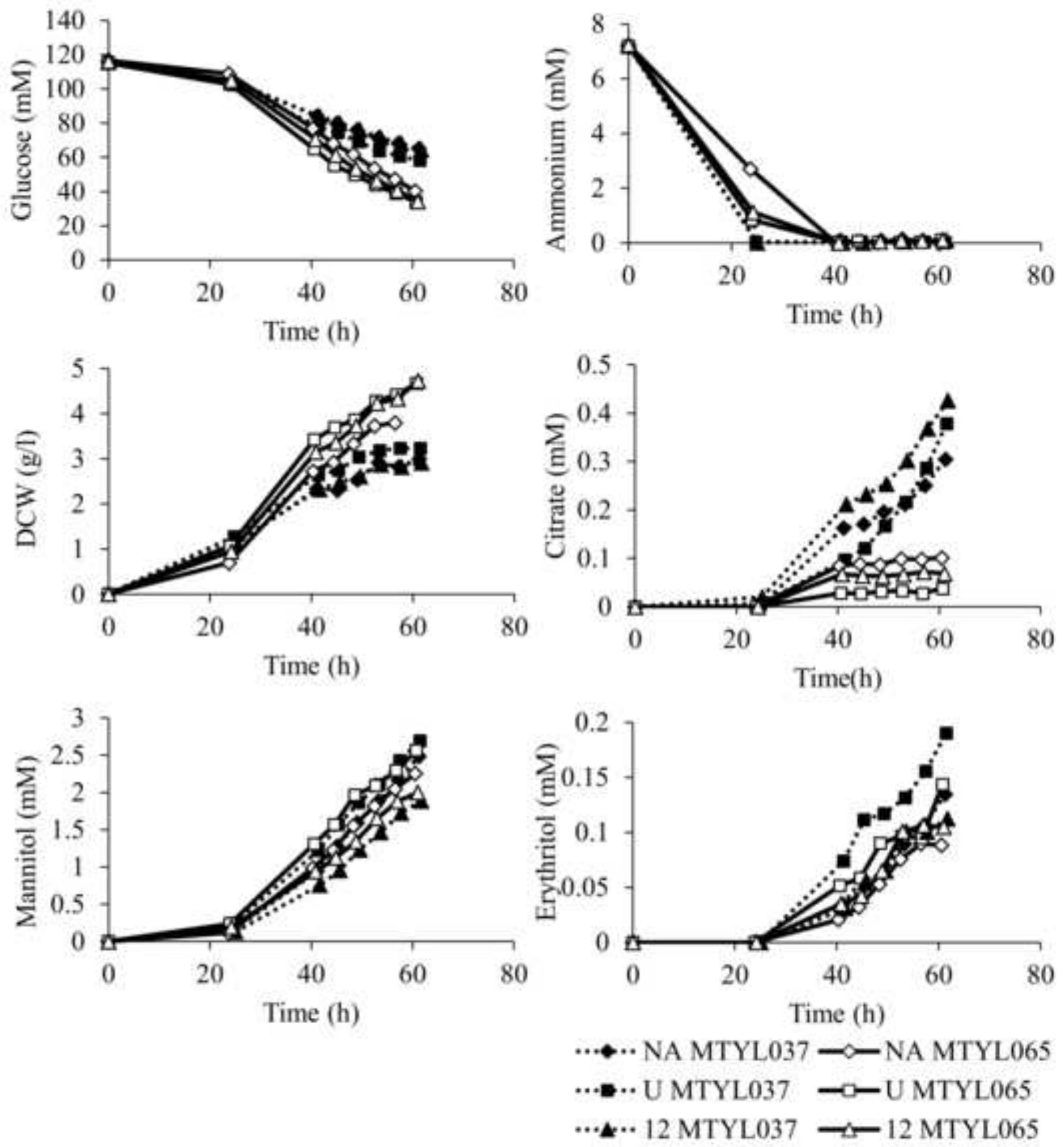

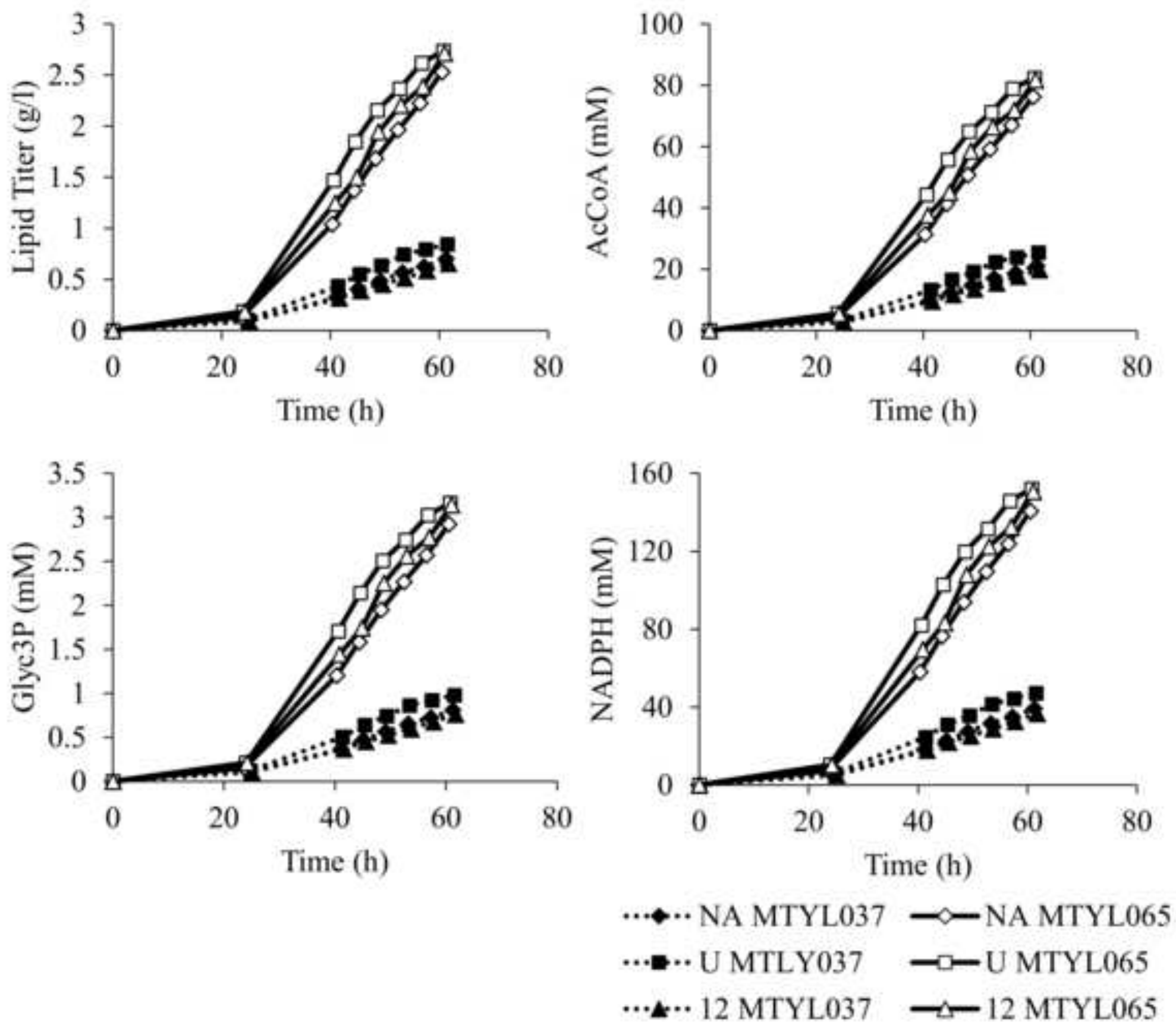


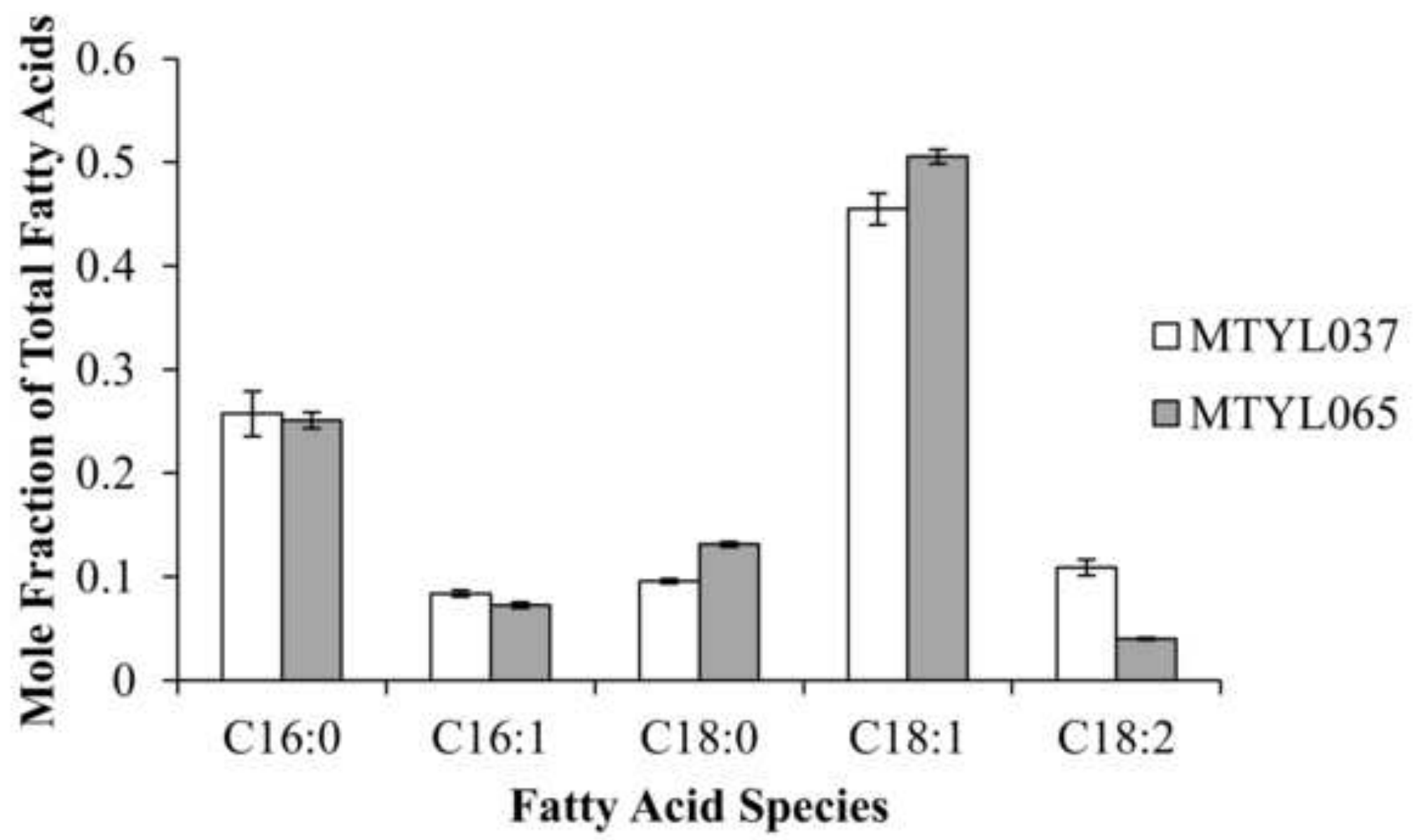


AcCoA $\rightarrow$ Lipids

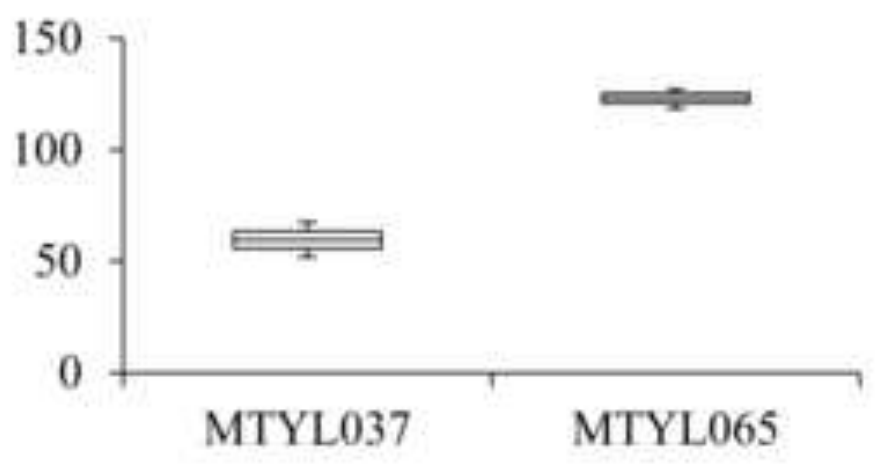

Malic Enzyme

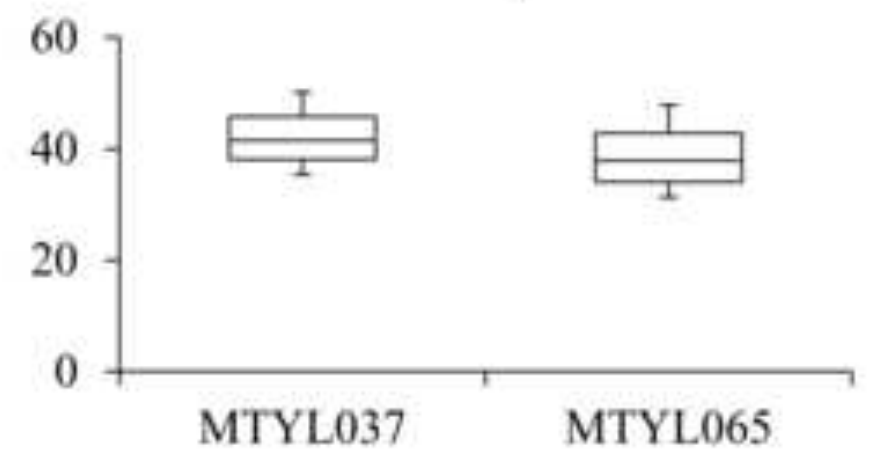

Oxidative PPP

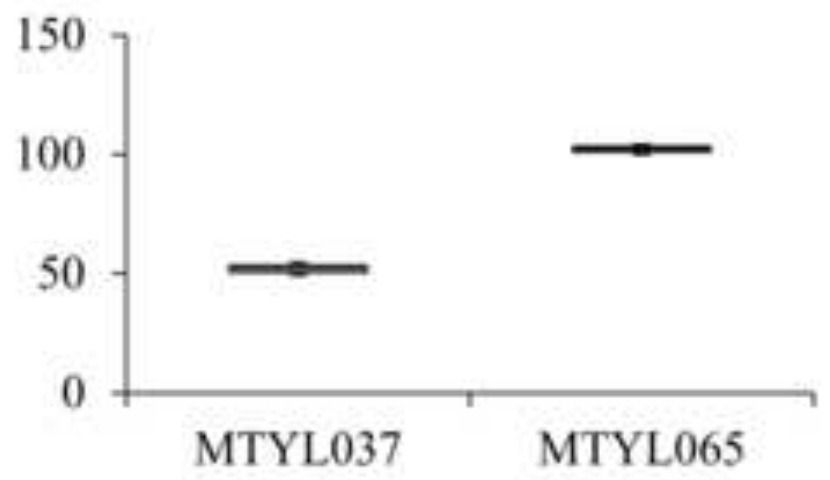

Phosphoglucose Isomerase

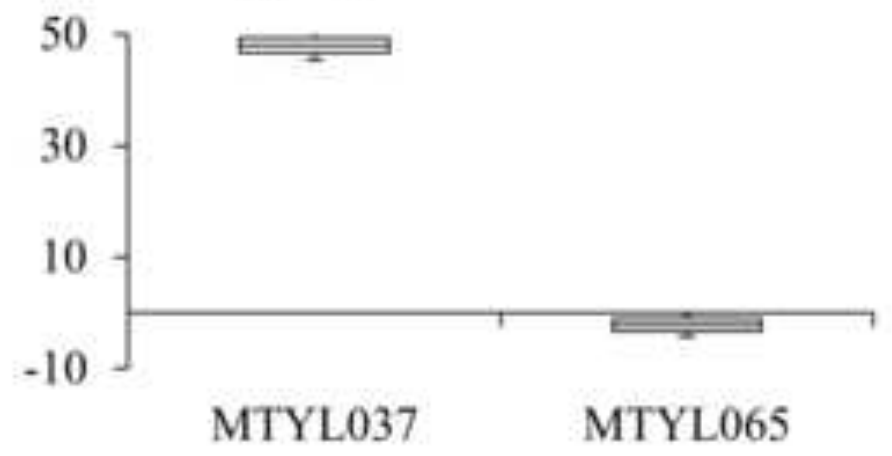

\section{Isocitrate Dehydrogenase}

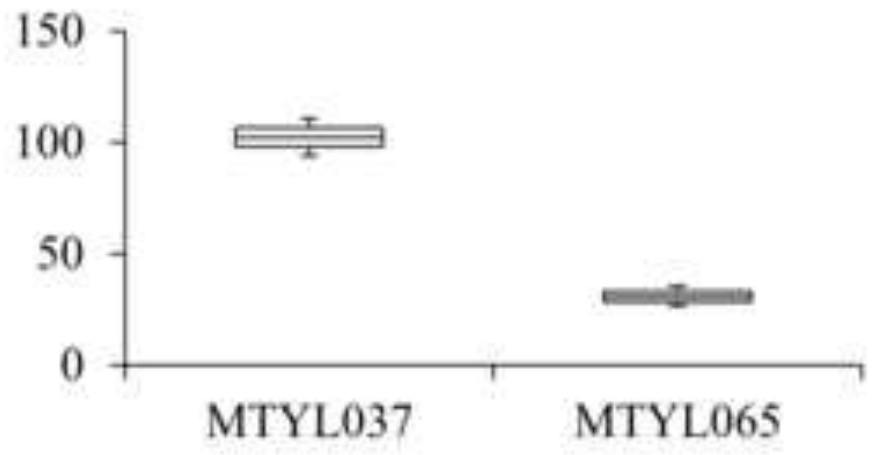


6PG

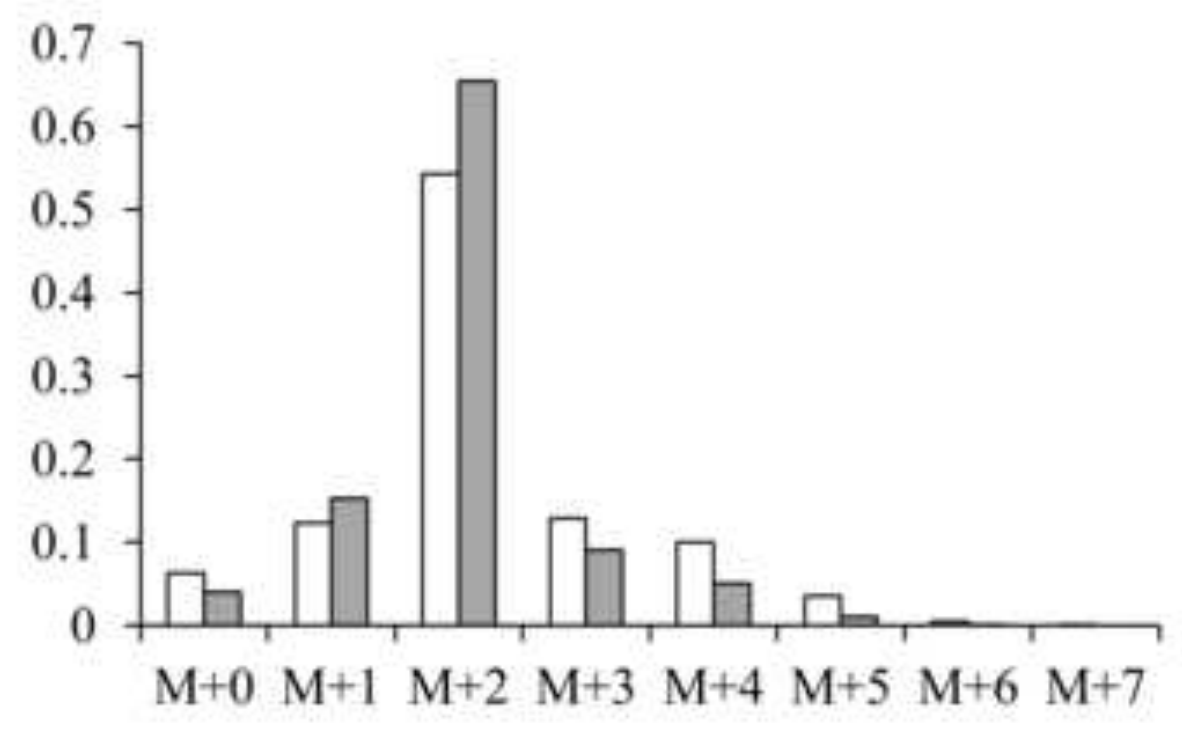

G6P

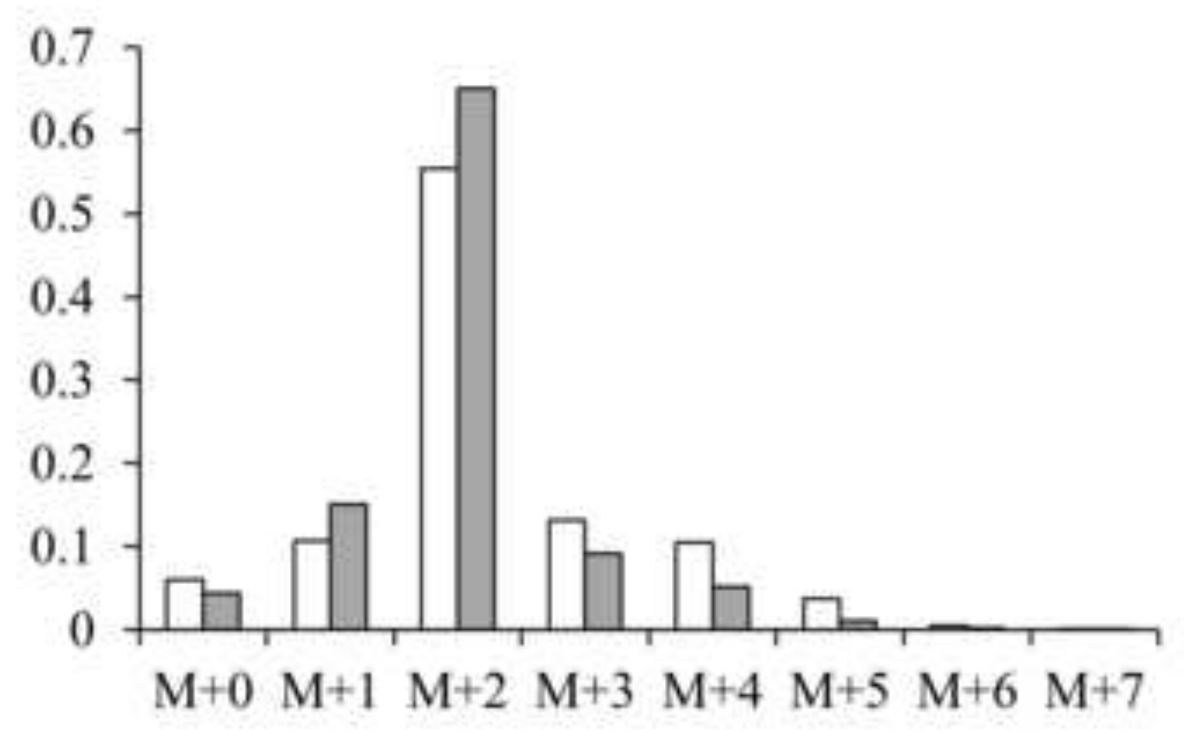

3PG

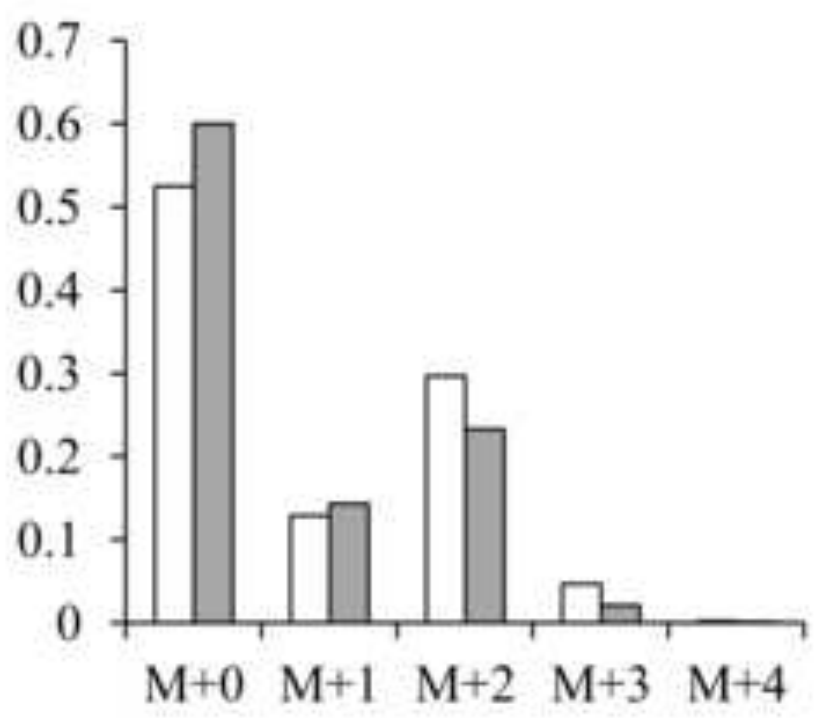

口MTYL037 口MTYL065

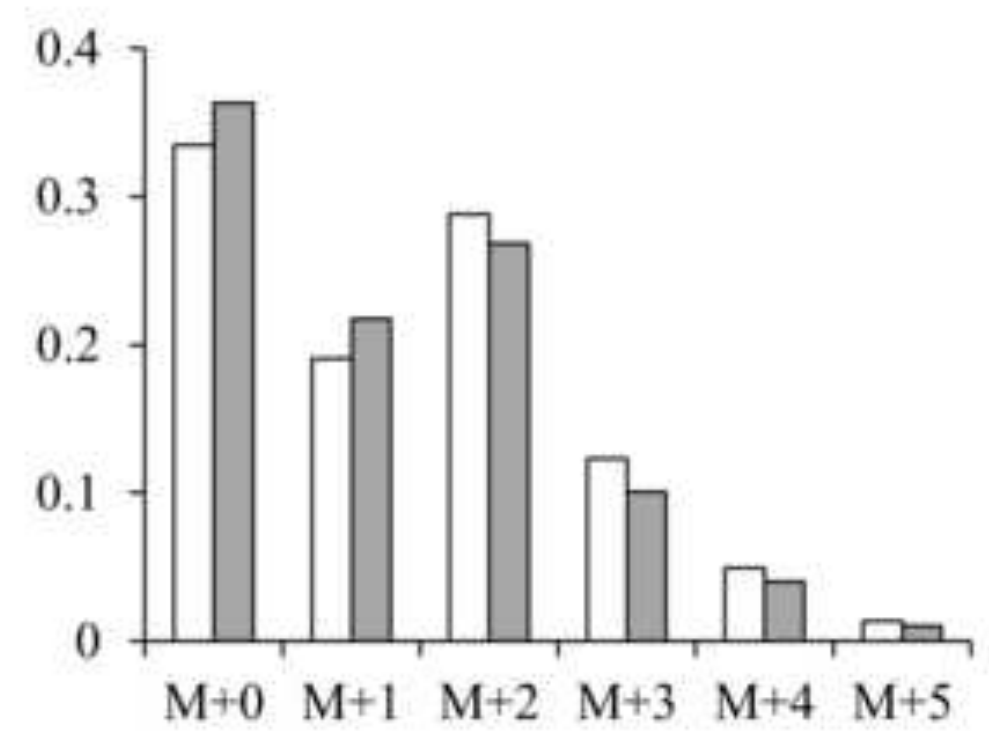

